\title{
Intrahepatic and intramyocellular lipids are determinants of insulin resistance in prepubertal children
}

\author{
D. E. Larson-Meyer • B. R. Newcomer • E. Ravussin • \\ J. Volaufova $\cdot$ B. Bennett $\cdot$ S. Chalew $\cdot$ W. T. Cefalu $~$ \\ M. Sothern
}

Received: 9 September 2010 /Accepted: 18 November 2010/Published online: 23 December 2010

(C) Springer-Verlag 2010

\begin{abstract}
Aims/hypothesis We hypothesised that ectopic fat deposition is present in liver and skeletal muscle before puberty and that both are potentially important factors in the early pathogenesis of insulin resistance.

Methods Proton magnetic resonance spectroscopy was used to evaluate intramyocellular and intrahepatic lipids in 50 male and 42 female multi-ethnic, prepubertal (Tanner $<2)$ children $(8.1 \pm 0.8$ years; $35.4 \pm 10.7 \mathrm{~kg} ; 27.9 \pm 8.3 \%$ body fat; means $\pm \mathrm{SD}$ ). Intramyocellular lipid was measured in soleus muscle and intrahepatic lipid in the middle right lobe. Abdominal fat was measured by magnetic resonance imaging, body fat by dual energy X-ray absorptiometry, and insulin resistance using homeostatic model assessment.
\end{abstract}

D. E. Larson-Meyer $(\square)$

Department of Family and Consumer Sciences

(Human Nutrition), Dept 3354, University of Wyoming,

1000 E, University Avenue,

Laramie, WY 82071, USA

e-mail: enette@uwyo.edu

B. R. Newcomer

Department of Clinical and Diagnostic Sciences,

University of Alabama,

Birmingham, AL, USA

J. Volaufova $\cdot$ B. Bennett $\cdot$ M. Sothern

School of Public Health, Louisiana State University

Health Sciences Center,

New Orleans, LA, USA

S. Chalew $\cdot$ W. T. Cefalu $\cdot$ M. Sothern

School of Medicine, Louisiana State University

Health Sciences Center,

New Orleans, LA, USA

E. Ravussin · J. Volaufova $\cdot$ W. T. Cefalu $\cdot$ M. Sothern

Pennington Biomedical Research Center,

Baton Rouge, LA, USA
Results Intrahepatic lipid ranged from $0.11 \%$ to $4.6 \%$ relative to the liver water signal (mean $0.79 \pm 0.79 \%$ ) whereas intramyocellular lipid ranged from $0.13 \%$ to $1.86 \%$ relative to the muscle water signal (mean $0.51 \pm$ $0.28 \%$ ). Intramyocellular and intrahepatic lipids were significantly correlated with total adiposity $(r=0.49$ and 0.59 ), abdominal adiposity ( $r=0.44$ and 0.54$)$, and each other $(r=0.39, p<0.05$, Spearman). Both intramyocellular and intrahepatic lipid were positively correlated with fasting insulin ( $r=0.37$ and 0.38 respectively) and insulin resistance $(r=0.37$ and $0.37 ; p<0.01)$. After adjustment for race and sex, the relations between ectopic fat and insulin resistance remained, whereas both disappeared when further adjusted for body fat or BMI $z$ scores.

Conclusions/Interpretations These results suggest that typical relations between body composition, ectopic fat and insulin resistance are present in children before puberty. Thus, interventions aimed at reducing adiposity have the potential to decrease ectopic fat accumulation, delay the onset of insulin resistance and decrease the risk for development of type 2 diabetes in children.

Keywords Diabetes in childhood · Human · Insulin sensitivity and resistance - Metabolic syndrome .

Pathophysiology/metabolism - Prediction and prevention of type 2 diabetes
Abbreviations
ALT Alanine amino transferase
AST Aspartate amino transferase
EMCL Extramyocellular lipid
${ }^{1} \mathrm{H}$ - Proton magnetic resonance spectroscopy
MRS
IHL Intrahepatic lipid
IMCL Intramyocellular lipid 
MET Mechanisms for the Metabolic Syndrome in Prepubertal Youth

MRI Magnetic resonance imaging

PRESS Point RESolved Spectroscopy

SAT Subcutaneous abdominal adipose tissue

TAT Total abdominal adipose tissue

VAT Visceral adipose tissue

\section{Introduction}

Insulin resistance is an early metabolic abnormality that precedes the development of glucose intolerance and overt type 2 diabetes [1]. It is hypothesised that the underlying pathology of insulin resistance is related to altered (or abnormal) partitioning of lipid between adipose and other tissues including skeletal muscle, liver, heart, pancreas and blood, i.e. ectopic fat stores [2]. One hypothesis is that abnormal partitioning may be triggered by a failure of subcutaneous adipocyte differentiation, which results in lipid overflow into visceral fat and non-adipose tissues [3]. Another hypothesis is that adipose tissue insulin resistance increases NEFA flux resulting from lipolysis, which in turn impairs insulin receptor signalling in liver and muscle [4]. In adults [5-10] and adolescents [4, 7, 11], accumulation of lipid within skeletal muscle (intramyocellular lipid [IMCL]) $[5-7,9,10]$ and liver (intrahepatic lipid [IHL]) $[4,8,11-$ 13] is associated with attenuation in insulin action. In adults, IHL has also been found to correlate with visceral adipose tissue (VAT) $[8,14]$ and enlarged adipocyte size [8].

It is not yet established whether metabolic antecedents of insulin resistance, including ectopic fat accumulation, occur in early childhood before puberty. Although studies in both deceased and overweight/obese children suggest that lipid accumulation in liver [15-18] or muscle [19] is initiated before puberty, it remains unclear whether ectopic fat accumulates similarly in liver and muscle tissues and whether it correlates with other markers of the metabolic syndrome and type 2 diabetes in both healthy non-obese and obese exclusively prepubertal children. Addressing this question is of importance given the increasing prevalence of insulin resistance and its related diseases in young children $[16,17,20,21]$. We previously reported that the assessment of IHL is feasible in healthy, prepubertal 7 9 year old children using proton magnetic resonance spectroscopy $\left({ }^{1} \mathrm{H}-\mathrm{MRS}\right)$, a non-invasive and safe procedure [18]. The purpose of the current study was to simultaneously evaluate IHL and IMCL stores in a healthy cohort of children prior to puberty, and correlate these ectopic lipid stores with insulin resistance and known components of the metabolic syndrome. We hypothesised that ectopic fat deposition is present in liver and skeletal muscle before puberty, and that both are potentially important factors in the early pathogenesis of insulin resistance.

\section{Methods}

Participants A total of 92 healthy children (7-9 years old) with complete IHL and IMCL data were included in the present analysis from the ongoing study Mechanisms of the Metabolic Syndrome in Prepubertal Youth (MET). MET is a cross-sectional study exploring markers for the metabolic syndrome in healthy, exclusively prepubertal youth. White, African-American, and other (Hispanic, Asian/Pacific Islander) children were recruited from south-east Louisiana, USA. Sixty-six per cent of the children enrolled into the study were recruited from area schools with the remainder coming from physicians' offices, informational mailings and health fairs. Prior to enrolment, medical and family histories were obtained through a detailed phone interview with the parents/guardians of interested volunteers. If eligible, a complete medical history, a physical examination including Tanner staging, and screening blood test (with comprehensive metabolic panel and complete blood count) were performed to confirm eligibility. Participants were excluded if they demonstrated evidence of significant cardiovascular disease, cardiac arrhythmia or liver disease, or chronically used medications, or had entered puberty (Tanner $\geq 2$ ); or if their mothers reported gestational diabetes or a family history (first degree relative) of type 1 or type 2 diabetes. The study was approved by the Institutional Review Board of Louisiana State University Health Sciences Center and Children's Hospital in New Orleans, the Pennington Biomedical Research Center and Woman's Hospital in Baton Rouge, LA, and the University of Wyoming. Participants' legal guardians read and signed an approved consent form and children gave their written assent prior to participation in any procedures.

Medical history, anthropometrics and blood sampling Participants included in this analysis were recruited from May 2006 to March 2010 and tested during one $2 \mathrm{~h}$ and one halfday outpatient visit at Children's Hospital and one half-day visit at Pennington Biomedical Research Center. Body weight was measured using an electronically calibrated scale (Indiana Scale, Terahuat, IN, USA) and height using a calibrated stadiometer (Holtain, Crymych, UK) without shoes according to standardised procedures; waist circumference was measured at the level of the umbilicus while standing; body composition was assessed by dual energy X-ray absorptiometry (Hologic QDR 4500A, Bedford, MA, USA; QDR for Windows Version 11.1.2); blood pressure was measured using a manual aneroid sphygmomanometer while participants were 
seated in a private, quiet examination room; insulin resistance was assessed by use of HOMA methodology [22]. Total abdominal adipose tissue (TAT) and VAT were measured by multi-slice magnetic resonance imaging (MRI); and IMCL and IHL stores were assessed using ${ }^{1} \mathrm{H}-\mathrm{MRS}$ [18]. A $12 \mathrm{~h}$ fasting blood sample was also drawn for analysis of insulin, glucose, liver function enzymes (alanine amino transferase [ALT] and aspartate amino transferase [AST]), cholesterol and triacylglycerol and a urine sample was obtained for ketone analysis. All blood chemistry and lipid analyses were performed on an Ortho Clinical Diagnostics VITROS 5,1 FS (Rochester, NY, USA), and LDL-cholesterol was calculated using the Friedewald equation.

MRI MRI scans were performed on a General Electric Sigma Excite (3.0 Tesla) whole body imaging and spectroscopy system (GE Medical Systems, Milwaukee, WI, USA). Children were placed on the scanner table in a hospital gown with hands over head. An eight channel torso-array coil was placed over the child's chest/abdomen area, and a series of scans from the highest point of the liver to the bottom pole of the right kidney were acquired. The images were analysed using the Analyze software package (CNSoftware, Rochester, MN, USA). The areas of TAT, VAT and subcutaneous abdominal adipose tissue (SAT) at approximately the L4-L5 disc space were calculated.

${ }^{1} H$-MRS IMCL and IHL were measured on the same Sigma Excite 3.0 Tesla system using the PRESS (Point RESolved Spectroscopy) technique. For muscle scans, children were positioned in the supine position with their knee in extension and ankle in a neutral position in the commercially provided knee coil as previously described [18]. Prior to positioning, the maximal circumference of the child's right calf was measured using a tape measure while standing bare-foot. This area was marked with a non-toxic marker and a vitamin E tablet was taped to the front side above the tibialis anterior muscle to identify the maximal cross-sectional area on the MRI scan. The area was then aligned with the centre of the coil. Rolled towels were positioned under the knee to provide support; foam was placed under the lower calf so that the tibialis was horizontal with the $\mathrm{B}_{0}$ magnetic field and the coil axis. Additional foam pads were positioned to fill the space in the knee coil; the free leg and coil were secured with a Velcro strap. Three independent non-overlapping watersuppressed PRESS voxels $\left(\sim 7 \times 7 \times 10 \mathrm{~mm}^{3}\right.$, echo time $=$ $35 \mathrm{~ms}$; repetition time $=1.5 \mathrm{~s}$; averages $=128$; reference averages $=16$ ) were collected from the largest crosssectional area of the soleus muscle (identified by the vitamin $E$ tablet on the scout images). Voxels were positioned as previously reported [18]. The reference spectra were extracted from the PRESS voxel data files to create a non-suppressed water spectrum for normalisation purposes. Finally, to increase the signal-to-noise of the spectra, PRESS voxels were summed before IMCL and extramyocellular lipid (EMCL) contents were determined in order to acquire a representative sampling of lipid content and optimise spectral quality, signal-to-noise ratio and total scan time relative to patient burden.

For liver scans, children were placed in a prone, feet-first orientation. After a preliminary localisation from an MRI scan, a single water-suppressed PRESS voxel $(\sim 3 \times 3 \times$ $3 \mathrm{~cm}^{3}$, echo time $=35 \mathrm{~ms}$, repetition time $=1.5 \mathrm{~s}$; number of averages $=128$, and 16 reference averages) and a single non-water suppressed PRESS voxel for normalisation were collected using the commercially provided ${ }^{1} \mathrm{H}$-body coil in an area of the middle right lobe that was free from heavy vascularisation as determined from the T1 weighted axial images ( 25 slices, $10 \mathrm{~mm}$ thick, $2 \mathrm{~mm}$ separation). These liver spectra represent an average liver fat measurement over the mid-right lobe because no respiratory gating was used. Liver motion was reduced by placement of the participant in the prone position, use of light restraints, and the practice of shallow breathing techniques [18]. To entertain the children and reduce any discomfort from loud noises, a personal music or story $C D$ was played via childsized headphones.

Peak positions were fit as previously described [8, 18]. Although all peaks were fit, the larger $\mathrm{CH}_{2}$ fatty acid peaks were selected to represent IHL, IMCL and EMCL. To improve reproducibility, an additional constraining factor was introduced to our previously published fitting model. This entailed fixing the line width of selected lipid peaks to the line width of the associated water peak for that voxel location. This enhanced prior knowledge fitting model proved to be the most reproducible and provided the least amount of residual signal after fitting. To account for dayto-day variation in system performance, IHL, IMCL and EMCL peak areas were expressed relative to the water signal from the non-water suppressed signal acquisition, which is assumed to be of constant concentration in this participant population. Finally all peaks of interest were corrected for $T_{1}$ and $T_{2}$ relaxation as well as normalised for PRESS voxel sizes and number of signal averages. The coefficient of variation for test-retest reliability of our methods at 3.0 Tesla is $9.36 \%$ for IHL, $8.35 \%$ for IMCL and $18.96 \%$ for EMCL.

Data analysis and statistical approach In the analysis we included 92 children with complete data on IHL and IMCL. Study data are not available for all children in the MET study due to occasional technical problems and/or the election of the child not to participate in this portion of the study, which was performed at the Pennington Biomedical Research Center, more than a $1 \mathrm{~h}$ drive away from New 
Orleans. Consequently, the sample size ( $n$ ) for all statistical analyses is reported in parentheses throughout the paper.

The data summarised in tables and text is presented as mean \pm SD. Associations between variables were determined by Spearman's Rank correlation coefficient due to the skewed distribution of some variables. Partial correlation coefficients were used to adjust for sex, race and either BMI $z$ scores or body fat $(\mathrm{kg})$. In all tests for zero correlation coefficient, the statistical significance was established at the 0.05 level. The analysis was performed using SAS Statistical software package Version 9.2.

\section{Results}

The characteristics of the 92 children enrolled in the study including their anthropometrics and fasting blood measurements are shown in Table 1. IHL stores were variable, ranging from $0.11 \%$ to $4.6 \%$ relative to the water peak (mean $0.79 \pm 0.79 \%$ ). Soleus IMCL ranged from $0.13 \%$ to $1.86 \%$ (mean $0.51 \pm 0.28 \%$ ) whereas soleus EMCL ranged from $0.25 \%$ to $4.3 \%$ (mean $1.1 \pm 0.73 \%$ ).

Liver and muscle lipid in relation to body size/composition IHL, IMCL and EMCL were correlated with body mass and markers of total body and central adiposity both before (data not shown) and after (Table 2) adjustment for sex and race. IMCL was also correlated with IHL $(r=0.39$, $p=0.0001)$ and EMCL $(r=0.24, p=0.02)$.

Liver and muscle lipid in relation to body insulin resistance IHL and IMCL were significantly $(p<0.01)$ correlated with fasting insulin concentration $(r=0.39$ and 0.43$)$ and insulin resistance ( $r=0.39$ and 0.43 ) before and after (Table 2) adjustment for sex and race. After further adjustment for BMI $z$ scores or body fat (data not shown), however, the relations between IHL and IMCL were not significant.

Relations between ectopic fat and markers associated with metabolic syndrome Six of a total of $62(9.7 \%)$ children with complete metabolic syndrome component data were positively diagnosed with metabolic syndrome based on the criteria reviewed by Golley et al. [23], which is specific to children aged 6-9 years [24]. Introducing waist circumference into the cluster required utilising another definition for metabolic syndrome, based on the European Group for the study of Insulin Resistance age adjusted criteria, and results were remarkably similar. Six of a total of 59 (10.1\%) children with waist circumference measures were identified as having metabolic syndrome [23]. When we examined the metabolic syndrome components independently we found that four of $64(6.2 \%)$ of the children had elevated total cholesterol ( $>5.5 \mathrm{mmol} / \mathrm{l})$ and five of $64(7.8 \%)$ had elevated fasting triacylglycerol $(>1.7 \mathrm{mmol} / \mathrm{l})$. Liver enzymes were also elevated in several participants. Seventeen of $86(19.8 \%)$ children had ALT concentrations higher than the upper limit of the normal range (5-30 U/l), and 10 of $88(11.4 \%)$ had AST concentrations above the upper limit $(>42 \mathrm{U} / 1)$. IHL showed significant $(p<0.05)$ correlations with systolic blood pressure $(r=0.28)$, total cholesterol $(r=0.35)$, LDL-cholesterol $(r=0.43)$ and fasting triacylglycerol concentrations $(r=0.26)$ but not HDLcholesterol $(r=-0.17)$, ALT $(r=0.19)$ and AST $(r=-0.13$; $n=88)$ concentrations, or diastolic blood pressure $(r=0.08$; $n=92$ ). As shown in Table 2, all of these relations, except fasting triacylglycerol concentrations, remained after adjusting for sex and race. However, after further adjustment for BMI $z$ scores or body fat either alone or in combination with sex and race, only the associations between IHL and total and LDL-cholesterol remained. In contrast, IMCL was significantly correlated only with systolic blood pressure before $(r=0.30)$ or after adjustment for sex and race (Table 2).

\section{Discussion}

This study used ${ }^{1} \mathrm{H}-\mathrm{MRS}$ to non-invasively measure lipid accretion in both liver and skeletal muscle in a large multiethnic cohort of healthy, exclusively prepubertal children, 7 9 years of age. These findings establish for the first time that many of the relations between ectopic fat and markers of the metabolic syndrome observed in adults are also present in healthy, young, non-diabetic children. Most noteworthy, we found that lipid accretion in liver and skeletal muscle is associated with fasting insulin and insulin resistance measured by HOMA. The relation between ectopic fat accumulation and insulin resistance, however, appears to be driven by increased total body and central adiposity. Our results suggest that excess adiposity (rather than maturation or puberty) promotes accumulation of lipid in liver and skeletal muscle, and that ectopic fat accumulation in both tissues may be important in the early pathogenesis of insulin resistance. To unravel the sequence of these complex mechanisms, including the inter-relation between abdominal fat, liver lipid accretion and insulin resistance, longitudinal studies in developing children are needed to delineate the time course of lipid accumulation in liver and within skeletal muscle in relation to insulin resistance.

The relation between total and abdominal adiposity and ectopic fat accumulation within skeletal muscle and liver in adults $[5,8,12]$ and within skeletal muscle in children [19] and adolescents [11] has previously been reported. The present study extends these findings to healthy, multi-ethnic, 
Table 1 Physical characteristics and fasting concentrations of glucose, insulin, cholesterol and liver enzymes in prepubertal children

\begin{tabular}{|c|c|c|c|}
\hline Variable & $n$ & Mean $\pm \mathrm{SD}$ or $n / n$ & Range \\
\hline \multicolumn{4}{|l|}{ Physical characteristics and anthropometrics } \\
\hline Sex ( $n$ male $/ n$ female) & 92 & $50 / 42$ & - \\
\hline Race ( $n$ non-white $/ n$ white) & 92 & $26 / 66$ & - \\
\hline Age (years) & 92 & $8.1 \pm 0.8$ & $7-9$ \\
\hline Height (cm) & 92 & $132.0 \pm 8.1$ & $110.7-156.5$ \\
\hline Body mass $(\mathrm{kg})$ & 92 & $35.4 \pm 10.7$ & $18.2-73.7$ \\
\hline BMI $\left(\mathrm{kg} / \mathrm{m}^{2}\right)$ & 92 & $20.4 \pm 4.6$ & $14.1-34.0$ \\
\hline BMI $z$ score (non-obese, $z$ score $<2 /$ obese, $z$ score $\geq 2$ ) & 92 & $68 / 24$ & - \\
\hline Body fat $(\%)$ & 92 & $27.9 \pm 8.3$ & $12.1-46.6$ \\
\hline Waist circumference $(\mathrm{cm})$ & 75 & $68.3 \pm 12.6$ & $48.0-102.1$ \\
\hline $\operatorname{TAT}\left(\mathrm{cm}^{2}\right)$ & 75 & $153 \pm 107.1$ & $23.7-478.2$ \\
\hline VAT $\left(\mathrm{cm}^{2}\right)$ & 75 & $13.3 \pm 7.1$ & $3.9-43.7$ \\
\hline SAT $\left(\mathrm{cm}^{2}\right)$ & 75 & $139.7 \pm 102.1$ & $19.7-461.4$ \\
\hline Diastolic blood pressure $(\mathrm{mmHg})$ & 92 & $64.3 \pm 5.5$ & $54.0-78.7$ \\
\hline Systolic blood pressure (mmHg) & 92 & $109.2 \pm 9.2$ & $85.5-135.3$ \\
\hline \multicolumn{4}{|l|}{ Fasting blood concentrations } \\
\hline Glucose $(\mathrm{mmol} / \mathrm{l})$ & 68 & $4.2 \pm 0.5$ & $1.83-5.3$ \\
\hline Insulin $(\mathrm{pmol} / \mathrm{l})$ & 63 & $31.6 \pm 30.9$ & $3.6-186.6$ \\
\hline Insulin resistance (HOMA) & 62 & $0.88 \pm 0.97$ & $0.07-6.03$ \\
\hline Total cholesterol $(\mathrm{mmol} / \mathrm{l})$ & 64 & $4.1 \pm 0.7$ & $2.7-5.9$ \\
\hline LDL-cholesterol (mmol/1) & 65 & $2.4 \pm 0.6$ & $1.2-4.1$ \\
\hline HDL-cholesterol (mmol/1) & 65 & $1.3 \pm 0.3$ & $0.7-2.2$ \\
\hline Triacylglycerol (mmol/1) & 64 & $0.9 \pm 0.6$ & $0.4-2.9$ \\
\hline ALT (U/1) & 86 & $27 \pm 8$ & $12-67$ \\
\hline AST (U/l) & 88 & $35 \pm 6$ & $24-50$ \\
\hline
\end{tabular}

exclusively prepubertal children, and demonstrates that ectopic fat accumulation in both liver and skeletal muscle is greater in those children with increased adiposity. Liver lipid stores, in particular, were strongly correlated with total body and abdominal visceral fat independent of sex and race. This association between IHL and total, but not necessarily visceral, abdominal fat is intriguing given that the majority of the abdominal fat deposition in these children was as SAT (91\% of total) rather than VAT (9\% of total; Table 1). Visceral and deep subcutaneous stores along with liver fat have been associated with metabolic derangements including insulin resistance in several studies [8, 12, 14, 25], but it has proven difficult to evaluate the independent effect of these depots. The tight association between liver fat and VAT is thought to be due to increased VAT-driven NEFA flux to the liver via portal circulation. Although we demonstrate similar relations in this cross-sectional study, longitudinal studies in prepubertal children will help resolve the inter-relations between these variables and determine which depot is initially most deleterious for insulin resistance and its associated metabolic complications.

A strong association between liver fat accumulation, or hepatic steatosis, and insulin resistance was previously identified in adults [8, 12, 14], adolescents [4, 11, 13] and obese prepubertal children [17]. The current study is the first to report such ectopic lipid accumulation in an ethnically diverse cohort of healthy primarily non-obese (73.9\%) children prior to puberty. The current findings are in support of a pilot study from our group using a clinical $1.5 \mathrm{~T}$ system [18] and the autopsy studies by Schwimmer and colleagues [15], which provided evidence that lipid can accumulate in liver at a young age. In the classic report of Schwimmer et al. [15], for example, autopsies of 742 deceased children were used to identify prevalence of fatty liver, which was $9.6 \%$ in children 2-19 years of age and $3.3 \%$ in those 5-9 years old. Clinical studies in severely obese children $[16,17]$ demonstrated that the prevalence of fatty liver or steatosis may be as high as $36-52 \%$ in obese paediatric patients, depending on Tanner stage and degree of obesity. The current study, which non-invasively measured IHL accumulation, extends previous findings and demonstrates that IHL accumulation in a multi-ethnic cohort of healthy, exclusively prepubertal children (range $0.11-4.6 \%$; mean $0.83 \%$ ) was similar to that found in healthy overweight, insulin sensitive adults (range 0.04 $7.8 \%$; mean $0.78 \%$ ) using the same methodology (E. 
Table 2 Relations between ectopic fat, total and central adiposity, blood pressure, insulin resistance, serum lipids and markers of liver function following adjustment for sex and race

\begin{tabular}{lllll}
\hline Variable & $n$ & IHL & IMCL & EMCL \\
\hline Body mass & 92 & $0.45^{*}$ & $0.41^{*}$ & $0.37^{*}$ \\
BMI & 92 & $0.55^{*}$ & $0.46^{*}$ & $0.34^{*}$ \\
BMI $z$ score & 92 & $0.56^{*}$ & $0.46^{*}$ & $0.31^{*}$ \\
Body fat & 92 & $0.59^{*}$ & $0.49^{*}$ & $0.42^{*}$ \\
Waist circumference & 75 & $0.42^{*}$ & $0.34^{*}$ & $0.40^{*}$ \\
TAT $\left(\mathrm{cm}^{2}\right)$ & 75 & $0.54^{*}$ & $0.44^{*}$ & $0.36^{*}$ \\
VAT $\left(\mathrm{cm}^{2}\right)$ & 75 & $0.43^{*}$ & $0.41^{*}$ & $0.33^{*}$ \\
SAT $\left(\mathrm{cm}^{2}\right)$ & 75 & $0.54^{*}$ & $0.43^{*}$ & $0.35^{*}$ \\
Diastolic blood pressure & 92 & 0.02 & 0.02 & -0.02 \\
Systolic blood pressure & 92 & $0.26^{*}$ & $0.32^{*}$ & 0.13 \\
Glucose & 68 & 0.12 & 0.07 & -0.03 \\
Insulin, fasting & 63 & $0.38^{*}$ & $0.37^{*}$ & 0.06 \\
Insulin resistance & 62 & $0.37^{*}$ & $0.37^{*}$ & -0.01 \\
Total cholesterol & 64 & $0.37^{*}$ & 0.19 & 0.09 \\
LDL-cholesterol & 65 & $0.42^{*}$ & 0.24 & 0.19 \\
HDL-cholesterol & 65 & -0.06 & -0.12 & -0.16 \\
Triacylglycerol & 64 & 0.21 & 0.17 & 0.22 \\
ALT & 86 & 0.17 & -0.02 & 0.07 \\
AST & 88 & -0.10 & -0.18 & -0.01 \\
\hline D & & & & \\
\hline & & & &
\end{tabular}

Data are partial correlation coefficients adjusted for sex and race ${ }^{*} p<0.05$ by Spearman's rank correlation procedures

Ravussin, unpublished observations) and that a greater accumulation of IHL was associated with insulin resistance, independent of sex and race.

We also found an association between IMCL, but not EMCL, and both fasting insulin and insulin resistance, as we had hypothesised. These results agree with the majority of studies in adults [5, 6, 9, 10], and adolescents [7] and one study in prepubertal boys [19]. This observation is of particular interest because our cohort, which was randomly recruited from South Louisiana, was comprised of predominantly non-obese male and female children with obesity prevalence rates slightly greater than reports in US youth [26]. In addition, children with a Tanner stage $\geq 2$ were excluded from enrolment, as were children with first degree relatives with diabetes. Furthermore, the range of IMCL in the soleus $(0.13-1.86 \%)$ was lower than previously found in adults $(0.1-3.5 \%)$ (E. Ravussin, unpublished observations). In two previous studies in 7-10 year old boys [19] and 11-15 year old adolescents [7], elevated total body fat and central adiposity were accompanied by higher IMCL [7, 19] and EMCL [7] depots. Our results in this exclusively prepubertal, healthy cohort of male and female children support these findings and suggest that IMCL and EMCL accumulate as a function of increasing obesity. Although EMCL also accumulated in association with increased adiposity, only IMCL appeared to be an important marker of elevated fasting insulin and insulin resistance in prepubertal youth, as in adults $[9,10]$. It is important to mention, however, that EMCL data are difficult to interpret because of the inhomogeneous storage of EMCL and methodological error (i.e. EMCL is susceptible to bulk magnetic susceptibility during assessment by MRS) [27].

A final noteworthy finding from our study was the strong relation between liver lipid accretion (and to some extent muscle lipid accretion) and components of the metabolic or insulin resistance syndrome [24]. In adults, the metabolic syndrome is defined as a cluster of metabolic abnormalities that predispose an individual to increased risk of cardiovascular disease and type 2 diabetes [28]. In children, especially prior to puberty, this definition is less clear. Using recent criteria specific to prepubertal youth, our study found that only six children $(9.7 \%)$ were positively diagnosed with the metabolic syndrome [23, 24]. We also found that elevated IHL was significantly correlated with several accepted characteristics of this metabolic cluster [28] including increased waist circumference, elevated systolic blood pressure and elevated fasting triacylglycerol concentration; but not with low HDL-cholesterol concentration, elevated fasting glucose concentration and/or elevated diastolic blood pressure. In addition to the standard characteristics, increased IHL was associated with increased total and LDL-cholesterol concentrations. Associations between increased liver fat accretion and many of these and other markers were previously reported in adolescent and obese children including elevated waist circumference [17], elevated waist-to-hip ratio [16], elevated fasting insulin [13] and triacylglycerol concentrations [13, 16, 17], and depressed HDL concentration [13, 16, 17], as well high normal to elevated liver enzyme concentrations [16, 17]. Nevertheless, the clustering of IHL with components of metabolic syndrome in healthy, primarily non-obese children prior to puberty is of clinical interest and may be used to help better define and detect metabolic syndrome in children under 10 years of age [20].

Overall the results of this study contribute to our understanding of the pathogenesis of insulin resistance in children before puberty. They suggest that increasing adiposity promotes ectopic fat accumulation in liver, but to a lesser degree in skeletal muscle, and that fat accumulation in liver and skeletal muscle are equally associated with insulin resistance. While these results imply that ectopic fat accumulation may be important in the early pathogenesis of insulin resistance, longitudinal studies are needed to help determine the cause and effect relationship for this association. However, our findings in this multiethnic cohort of healthy male and female children prior to puberty have significant clinical relevance, as interventions aimed at reducing adiposity have the potential to prevent or 
decrease ectopic fat accumulation and, thus, delay the onset of insulin resistance and the consequent risk for development of type 2 diabetes.

Acknowledgements We would like to acknowledge the many physicians, nurses, technicians and coordinators who were responsible for clinical assessments and data collection at the Louisiana State University Health Sciences Center School of Public Health and Department of Pediatrics, the Children's Hospital of New Orleans, the Pennington Biomedical Research Center and Woman's Hospital of Baton Rouge. This work was supported by grants from the National Institute of Child Health \& Human Development R01 HD41071-01A2 entitled 'Insulin Sensitivity in African-American and Caucasian Youth with Low Birth' and R01 HD49046 entitled 'Mechanisms of the Metabolic Syndrome in Prepubertal Youth'. It was also supported in part by the Louisiana State University Health Sciences Center and Tulane University's Clinical and Translational Research Center as part of the Louisiana Board of Regents RC/EEP Fund, and funded in part by the Clinical Nutrition Research Unit Grant to ER \# 1P30 DK072476 entitled 'Nutritional Programming: Environmental and Molecular Interactions'.

Duality of interest The authors declare that there is no duality of interest associated with this manuscript.

\section{References}

1. Reaven GM (1988) Banting lecture 1988. Role of insulin resistance in human disease. Diabetes 37:1595-1607

2. Shulman GI (2000) Cellular mechanisms of insulin resistance. J Clin Invest 106:171-176

3. Danforth E Jr (2000) Failure of adipocyte differentiation causes type II diabetes mellitus? Nat Genet 26:13

4. Fabbrini E, deHaseth D, Deivanayagam S, Mohammed BS, Vitola BE, Klein S (2009) Alterations in fatty acid kinetics in obese adolescents with increased intrahepatic triglyceride content. Obesity (Silver Spring) 17:25-29

5. Forouhi NG, Jenkinson G, THomas EL et al (1999) Relation of triglyceride stores in skeletal muscle cells to central obesity and insulin sensitivity in European and South Asian men. Diabetologia 42:932-935

6. Perseghin G, Scifo P, de Cobelli F et al (1999) Intramyocellular triglyceride content is a determinant of in vivo insulin resistance in humans: a $1 \mathrm{H}-13 \mathrm{C}$ nuclear magnetic resonance spectroscopy assessment in offspring of type 2 diabetic parents. Diabetes 48:1600-1606

7. Sinha R, Dufour S, Petersen KF et al (2002) Assessment of skeletal muscle triglyceride content by (1)H nuclear magnetic resonance spectroscopy in lean and obese adolescents: relationships to insulin sensitivity, total body fat, and central adiposity. Diabetes 51:1022-1027

8. Larson-Meyer DE, Heilbronn LK, Redman LM et al (2006) Effect of calorie restriction with or without exercise on insulin sensitivity, beta-cell function, fat cell size, and ectopic lipid in overweight subjects. Diabetes Care 29:1337-1344

9. Krssak M, Falk Petersen F, Dresner A et al (1999) Intramyocellular lipid concentrations are correlated with insulin sensitivity in humans: a $1 \mathrm{H}$ NMR spectroscopy study (rapid communication). Diabetologia 42:113-116

10. Jacob S, Machann J, Rett K et al (1999) Association of increased intramyocellular lipid content with insulin resistance in lean nondiabetic offspring of type 2 diabetic subjects. Diabetes 48:1113-1119
11. Perseghin G, Bonfanti R, Magni S et al (2006) Insulin resistance and whole body energy homeostasis in obese adolescents with fatty liver disease. Am J Physiol Endocrinol Metab 291:E697E703

12. Ryysy L, Hakkinen AM, Goto T et al (2000) Hepatic fat content and insulin action on free fatty acids and glucose metabolism rather than insulin absorption are associated with insulin requirements during insulin therapy in type 2 diabetic patients. Diabetes 49:749-758

13. Cali AM, Zern TL, Taksali SE et al (2007) Intrahepatic fat accumulation and alterations in lipoprotein composition in obese adolescents: a perfect pro-atherogenic state. Diabetes Care 30:3093-3098

14. Fabbrini E, Magkos F, Mohammed BS et al (2009) Intrahepatic fat, not visceral fat, is linked with metabolic complications of obesity. Proc Natl Acad Sci USA 106:15430-15435

15. Schwimmer JB, Deutsch R, Kahen T, Lavine JE, Stanley C, Behling C (2006) Prevalence of fatty liver in children and adolescents. Pediatrics 118:1388-1393

16. Guzzaloni G, Grugni G, Minocci A, Moro D, Morabito F (2000) Liver steatosis in juvenile obesity: correlations with lipid profile, hepatic biochemical parameters and glycemic and insulinemic responses to an oral glucose tolerance test. Int J Obes Relat Metab Disord 24:772-776

17. D'Adamo E, Impicciatore M, Capanna R et al (2008) Liver steatosis in obese prepubertal children: a possible role of insulin resistance. Obesity (Silver Spring) 16:677-683

18. Larson-Meyer DE, Newcomer BR, VanVrancken-Tompkins CL, Sothern M (2010) Feasibility of assessing liver lipid by proton magnetic resonance spectroscopy in healthy normal and overweight prepubertal children. Diabetes Technol Ther 12:207-212

19. Ashley MA, Buckley AJ, Criss AL et al (2002) Familial, anthropometric, and metabolic associations of intramyocellular lipid levels in prepubertal males. Pediatr Res 51:81-86

20. Zimmet P, Alberti G, Kaufman F et al (2007) The metabolic syndrome in children and adolescents. Lancet 369:2059-2061

21. Kaufman FR (2002) Type 2 diabetes mellitus in children and youth: a new epidemic. J Pediatr Endocrinol Metab 15(Suppl 2):737-744

22. Cutfield WS, Jefferies CA, Jackson WE, Robinson EM, Hofman PL (2003) Evaluation of HOMA and QUICKI as measures of insulin sensitivity in prepubertal children. Pediatr Diabetes 4:119125

23. Golley RK, Magarey AM, Steinbeck KS, Baur LA, Daniels LA (2006) Comparison of metabolic syndrome prevalence using six different definitions in overweight pre-pubertal children enrolled in a weight management study. Int J Obes (Lond) 30:853-860

24. Lambert M, Paradis G, O'Loughlin J, Delvin EE, Hanley JA, Levy E (2004) Insulin resistance syndrome in a representative sample of children and adolescents from Quebec, Canada. Int J Obes Relat Metab Disord 28:833-841

25. Smith SR, Lovejoy JC, Greenway F et al (2001) Contributions of total body fat, abdominal subcutaneous adipose tissue compartments, and visceral adipose tissue to the metabolic complications of obesity. Metabolism 50:425-435

26. Ogden CL, Carroll MD, Curtin LR et al (2010) Prevalence of high body mass index in US children and adolescents, 2007-2008. JAMA 303(3):242-249

27. Boesch C, Slotboom J, Hoppeler H, Kreis R (1997) In vivo determination of intra-myocellular lipids in human muscle by means of localized ${ }^{1} \mathrm{H}-\mathrm{MR}$-spectroscopy. Magn Reson Med $37: 484-493$

28. NCE (2001) Executive summary of the third report of the National Cholesterol Education Program (NCEP) expert panel on detection, evaluation, and treatment of high blood cholesterol in adults (Adult Treatment Panel III). JAMA 285:2486-2497 